\title{
Aula invertida para la mejora del aprendizaje en la asignatura de Transferencia de Calor y Materia Flip Teaching for the improvement of learning in the subject of Heat and Mass Transfer
}

\author{
Ricardo Castedo ${ }^{1}$, Lina M Mópez $^{1}$, Marcelo F. Ortega ${ }^{2}$, José D. Cabrera ${ }^{1}$, María J. García-Martínez², José A. \\ Sanchidrián ${ }^{1}$, Pablo Segarra ${ }^{1}$, Carlos Paredes ${ }^{1}$ \\ ricardo.castedo@upm.es, lina.lopez@upm.es, marcelo.ortega@upm.es,jd.cabrera@alumnos.upm.es,mj.garcia@upm.es, \\ ja.sanchidrian@upm.es, pablo.segarra@upm.es, carlos.paredes@upm.es \\ ${ }^{1}$ Departamento de Ingeniería Geológica y Minera \\ Universidad Politécnica de Madrid \\ Madrid, España \\ ${ }^{2}$ Departamento de Energía y Combustibles \\ Universidad Politécnica de Madrid \\ Madrid, España
}

\begin{abstract}
Resumen- Se ha desarrollado y aplicado la metodología Flip Teaching (aula invertida) en la asignatura obligatoria "Transferencia de Calor y Materia” de $2^{\circ}$ curso del grado de Ingeniero de la Energía en la ETSI de Ingenieros de Minas de la Universidad Politécnica de Madrid. Se tienen dos grupos, uno de control y otro experimental. El material que dispone el alumno preparado por el profesor para ambos grupos es: presentaciones de power point, el libro de texto y repositorio de problemas. El grupo experimental, además dispone de vídeos cortos colgados en Youtube y cuestionarios en Moodle. Estas actividades se integran en el aula con la explicación por parte del profesor de los errores detectados en los cuestionarios, y además los alumnos realizan ejercicios de aplicación de lo estudiado en casa. El efecto de la metodología se mide en el impacto del aprendizaje en los exámenes parciales de dos bloques de la asignatura (conducción y convección) que suponen el $66 \%$ de la misma. El resultado de la comparación es que las notas medias del grupo experimental son ligeramente mayores que el grupo de control. Así mismo, la dispersión de las notas es menor asegurando un mayor nivel del alumnado.
\end{abstract}

Palabras clave: Clase inversa, aprendizaje basado en problemas, tasas de asistencia, transferencia de calor y materia.

Abstract- A Flip Teaching methodology has been developed and applied into an obligatory subject of the 2nd curse titled "Heat and Mass transfer" of the Energetic Engineering Bachelor at the School of Mines and Energy at the Universidad Politécnica de Madrid. There are two groups, the control and the experimental one. To do that, both groups has a material prepared by the teacher such as power point presentations, a textbook, and problem repository. The experimental group, also has short videos on Youtube, and short tests on Moodle. These activities are integrated in the class with the explanation by the teacher of the errors detected in the tests, and the students made exercises of application. The effect of the methodology is measured in the learning impact through the partial exams of two parts (conduction and convection) of the subject which account for the $66 \%$ of it. The result is that the mean value of the grades are higher for the group that has this methodology than the control group. Also, the dispersion of the notes is less ensuring a better level of students.

Keywords: Flip teaching, problem-based learning, class attendance ratio, heat and mass transfer.

\section{INTRODUCCIÓN}

El modelo tradicional de enseñanza en las diferentes asignaturas de los primeros cursos de ingeniería se basa en impartir la lección magistral, donde el profesor habla o recita, $\mathrm{y}$ en el mejor de los casos el alumno escucha. Algunos profesores combinan esto con el envío de trabajos para casa, que luego ni son evaluados ni son tomados en cuenta. Basado en la idea de Confucio "Dime algo y lo olvidaré, enséñame algo y lo recordaré, hazme participe de algo y lo aprenderé!" aparecen numerosas técnicas para mejorar la clase tradicional. Desde el año 2000, aparece un cambio de modelo acuñado por Lage y Baker y que se conoce como "Flipped Classroom", "Flipped Learning" o "Flip Teaching" (Sein-Echaluce, Fidalgo-Blanco y García-Peñalvo, 2015).

El modelo de aula invertida se basa en que las fases receptivas del alumno (Soler et al., 2002): receptiva, reflexiva y retentiva; se realicen de manera independiente al ritmo en que el alumno considere efectivo. En estas fases el alumno no está solo y cuenta en general con estímulos audiovisuales, así como apuntes y/o ejercicios sencillos. Las fases reactivas (Soler et al., 2002): creativa, expresiva y práctica; las debe realizar en el aula con la guía y supervisión del profesor de manera individual y/o cooperativa.

Para que este modelo resulte efectivo de cara al aprendizaje, es bien conocido, que se requiere un elevado compromiso del alumno con su propio aprendizaje, así como, de un gran esfuerzo por parte del profesorado para transformar las materias clásicas en un aula invertida (Coates, 2006). Sin embargo, no existe ninguna uniformidad en su aplicación, ni en las técnicas empleadas, ni en los resultados obtenidos tanto en el aula como en el trabajo autónomo (O'Flaherty y Phillips, 2015). Además, existe un déficit en el análisis del impacto real de estas técnicas en el aprendizaje del alumno. En la mayoría de los estudios se analizan los resultados en base a cuestionarios de satisfacción tipo SEEQ o sólo en partes de la materia y no en el global de la misma (Sein-Echaluce, Fidalgo-Blanco y García-Peñalvo, 2015; Yoshida, 2016). 
Este trabajo presenta la total aplicación del modelo de Aula Invertida para una asignatura obligatoria de $2^{\circ}$ curso de ingeniería, tradicionalmente presencial pero con una alta carga de realización de problemas / ejercicios. Dicha asignatura ya estaba dividida en sesiones estructuradas, y por tanto, el esfuerzo del profesorado ha sido básicamente la realización de videos y la separación de las presentaciones por sesiones. Dicho esto, este trabajo persigue: a) disminuir el absentismo en clase (debido a los horarios a los que nos enfrentamos) y aumentar el porcentaje de presentados a los exámenes; b) conocer los hábitos de conectividad de los alumnos con los recursos (videos Youtube); c) medir el impacto del aula invertida en el aprendizaje en base al resultado en los exámenes parciales y/o finales de la asignatura; d) el uso de una herramienta de conexión recursos web-aula.

\section{CONTEXTO}

En los primeros cursos de las materias de cualquier ingeniería, los alumnos vienen con una base académica, una predisposición y una actitud muy dispar. La finalidad fundamental de la metodología desarrollada y aplicada es mejorar el proceso de aprendizaje del alumnado y de algunas competencias transversales. Para ello, decidimos emplear la técnica de aula inversa, combinada con actividades enlace basadas en cuestionarios y con trabajos cooperativos en el aula donde el profesor se integra con los alumnos en la resolución de los problemas.

El presente trabajo se ha desarrollado en la asignatura “Transferencia de Calor y Materia”, de la titulación Grado de Ingeniero de la Energía de la ETSI Minas y Energía de la Universidad Politécnica de Madrid en el curso 16-17. La asignatura es parte del segundo semestre del segundo curso, consta de 6 créditos y es de carácter obligatorio. La nota media de corte con la que esta promoción accedió al grado fue de 9,051 (curso 2015-16).

En el presente curso (2016-17), se tienen dos grupos, el grupo 1 (GIE1) con 72 alumnos y el grupo 2 (GIE2) con 80 alumnos. El grupo 1 tiene clase los lunes (de $12: 10$ a 14:00) y jueves (de 10:00 a 11:50), mientras que el grupo 2 tiene las clases los martes (de 12:10 a 14:00) y viernes (de 08:30 a 10:20). Debido al horario se elige el GIE2 como grupo experimental, para ver si a pesar de tener un horario en el que tradicionalmente asisten menos alumnos a clase, este efecto se ve mitigado de alguna manera con una metodología que requiera de su mayor implicación.

La materia está dividida en 4 partes, que en orden cronológico son: conducción (20 h - las 9 primeras clases), convección (20 h - de la clase 10 a la 18), transferencia de masa (14 h - de la clase 19 a la 24) y radiación (6 - de la 25 a la 27). Entre paréntesis se muestran las horas de clase en aula previstas para cada una de las partes. La asignatura comenzó en Febrero de 2017 y termina en Mayo de 2017, teniendo el examen final en Junio y el extraordinario en Julio de 2017. Las tres primeras partes tienen un examen liberatorio de evaluación continua. Mientras que la última se evalúa el mismo día del examen final de junio. Los resultados que aquí se presentan y analizan corresponden a las dos primeras partes de la materia: conducción y convección. Estas partes forman el $66 \%$ del contenido y por ende, de la evaluación de la asignatura.
En este proyecto se pretenden abarcar dos tipos de objetivos. Por un lado, tenemos los objetivos enfocados desde un punto de vista del profesorado, y por otro, los objetivos desde un punto de vista del desarrollo del alumno.

Por parte del profesorado:

- Se pretende promover la utilización de recursos tecnológicos como videos y cuestionarios de Moodle que nos permitan detectar carencias, y por tanto, mejorar la diversidad y carácter de los materiales educativos.

- Mejorar las tasas de éxito y reducir el posible absentismo del alumnado en las clases y en los exámenes, sin la necesidad de recurrir a dar puntos extra por asistencia.

\section{- Fomentar el trabajo continuo no presencial.}

- Determinar el tiempo empleado en la visualización de los videos y los soportes empleados para su visualización (de alguna manera determinar el grado de m-learning de nuestros estudiantes). Esto es importante, puesto que, a mayor desarrollo, mayor variedad de herramientas para combatir los problemas del sector educativo (UNESCO, 2013).

Por parte del alumnado:

- Contribuir al aprendizaje autónomo y flexible, basado en problemas, puesto que la base del conocimiento (lo que viene en los libros) lo debe adquirir el alumno de manera autónoma.

- Mejorar la concepción del alumno de su trabajo en casa y participación activa como parte fundamental de su propio aprendizaje.

- Por último y de algún modo incluida en las anteriores, el desarrollo y evaluación de competencias transversales como: organización y planificación, trabajo en equipo, uso de las TIC, resolución de problemas y en algunos casos liderazgo.

\section{DESCRIPCIÓN}

Las partes de la asignatura que se describen y posteriormente analizan son las de conducción y convección. Ambas están impartidas por diferentes profesores, por lo que de alguna manera se asegura una diversidad en los estímulos y en la forma de transmitir la materia al alumnado. Sin embargo, la estructura y metodología seguida por ambos profesores para ambos módulos es básicamente la misma (Figura 1).

\section{A. Test control homogeneidad}

Después de la primera sesión magistral realizada en ambos grupos (GIE1 y GIE2) con los mismos materiales y el mismo profesor, se realizó en la segunda sesión un cuestionario tipo test con 5 preguntas sobre cuestiones generales de la materia vistas en la primea sesión. Sólo se realizó una vez en la segunda sesión de conducción. Este cuestionario se realizó con el fin de analizar la homogeneidad de ambos grupos y establecer una base con la que analizar los resultados posteriores. Este tipo de pruebas se basan en las ideas de Mason, Shuman y Cook (2013) que analizan la homogeneidad de los grupos en diferentes materias o Fidalgo-Blanco, Martinez-Nuñez, Borrás-Gene y Sanchez-Medina (2017), que la analizan en base a tareas, empleo de foros y solución de problemas. 


\section{B. Recursos y tareas previas al aula con Moodle y Youtube}

En esta parte del aula invertida a cada alumno del grupo experimental (GIE2) se le proporciona el siguiente material a través de Moodle:

- Libro de consulta en castellano (Sanchidrián, 2014) que incluye los temas de conducción y convección con ejercicios propuestos (disponible en pdf).

- Presentaciones de power point realizadas para cada sesión, es decir, un resumen de los conceptos más importantes que vienen desarrollados en el libro. Es un material resumen que bien puede servir para estudiar antes incluso del examen. Se ha realizado una presentación por cada sesión de aula invertida, en total 8 para convección y 8 para conducción.

- Videos de entre 5 y 10 minutos, aunque siempre inferior a este último número, pues es sabido que a partir de ese rato el alumno deja de prestar atención (Medina, 2008). Estos videos combinan la imagen del profesor sobre las presentaciones de power point. Se ha realizado un video por cada sesión de aula invertida, en total 8 para convección y 8 para conducción. Estos vídeos se han subido en la plataforma Youtube de la que podemos extraer infinidad de información.

- Cuestionario de 5 preguntas, mezclando respuestas múltiples o verdadero/falso, obligatorio y por saturación. En estos cuestionarios el alumno debe obtener un 10, teniendo la posibilidad de realizar múltiples intentos sin penalización. Cuando el alumno obtiene un 10, el cuestionario de la sesión terminada le sirve de llave para el material de la siguiente clase o clases, y así sucesivamente.

- Colección de problemas resueltos que los alumnos encuentran disponible al llegar a la última clase del Bloque de la asignatura.

Por otra parte, el material disponible en Moodle para el grupo de control (GIE 1) desde el inicio del curso es: libro en pdf, presentaciones en Power Point de la asignatura y colección de problemas resueltos. Cabe destacar que al final de cada bloque, los alumnos de ambos grupos dispondrán de los mismos contenidos básicos (libro, presentaciones y colección de problemas resueltos), con el fin de no dar ventaja en ese sentido a ninguno de los grupos.

\section{Actividad nexo entre trabajo en casa - aula}

Esta actividad se puede realizar de diversas maneras según diferentes autores (Fidalgo-Blanco, Martinez-Nuñez, BorrásGene y Sanchez-Medina, 2017; Yoshida, 2016). En este curso, se disponía de un foro de profesores-alumnos, pero su uso ha sido residual posiblemente por la falta de dinamización por parte del profesorado, que a su vez desemboca en el olvido para los alumnos. En previsión de este resultado, se optó por la explicación por parte del profesorado de las preguntas con mayor porcentaje de fallo en los cuestionarios de Moodle. De tal manera que los 10 primeros minutos de cada clase el profesor los dedica a resolver las cuestiones que parecen estar peor entendidas por los alumnos.

\section{Actividad en el aula}

En el grupo de control o GIE1, en cada una de las sesiones presenciales de 100 minutos de duración, se explica la teoría y se resuelven problemas, aunque debido al tiempo disponible ambas tareas son llevadas a cabo por el profesor con la mínima participación e implicación activa del alumnado.
En el grupo experimental o GIE2, la clase impartida se reduce a 80 minutos (incluyendo los 10 de la actividad nexo) y se dedican exclusivamente a la realización de problemas y/o ejercicios. Los otros 20 minutos es lo que hemos estimado en ver el vídeo y realizar los cuestionarios. En estos casos, el profesor lanza la cuestión a los alumnos, que divididos en grupos aleatorios (no se sigue un orden o disciplina, cada alumno se sienta con quien quiere), realizan los ejercicios. Los grupos que terminan en primer lugar, salen a la pizarra y explican a sus compañeros el trabajo realizado. El papel del profesor es de guía o “compañero” (Cortés Martín, 2010) para resolver las dudas del alumno en la fase reactiva que no tanto dudas de la fase receptiva (dudas que debían estar resueltas entre vídeos, libro y actividades nexo). Además en este grupo, la última sesión de los dos bloques se emplea como repaso/dudas/ejercicios de todo lo realizado durante las 8 sesiones anteriores.

\section{Trabajo en casa}

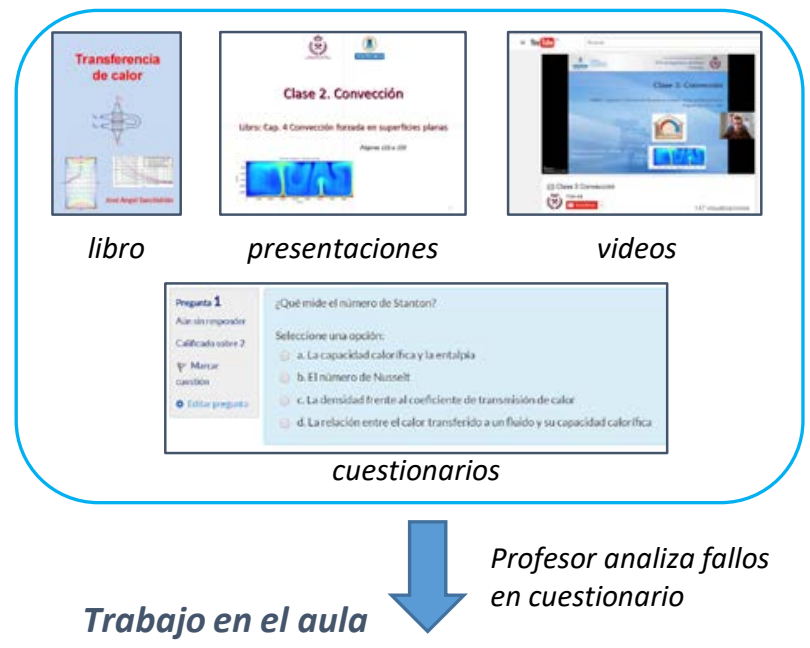

1. Explicación de los fallos más

frecuentes en los cuestionarios ( \pm 10 minutos)

\section{Trabajo en grupo para la} resolución de problemas/ejercicios.

\section{Resolución en común de los ejercicios. Salen a la pizarra alumnos.}

Figura 1. Diagrama de flujo de la metodología empleada.

\section{E. Examen de evaluación}

Por último, pero no menos importante, tenemos la evaluación. Ésta se realiza en base a exámenes parciales liberatorios. Es decir, todo alumno que tenga más de un 3 en un examen, puede no presentarse al final siempre que la media ponderada con el resto de partes dé como resultado un aprobado ( $>5$ sobre 10). Los resultados aquí presentados se basan en los exámenes de conducción y convección:

- Conducción: el examen consta de 3 preguntas teóricoprácticas donde el alumno puede llevar un resumen en papel de lo que considere importante de hasta 3 caras, sin ejercicios. Las clases de este bloque terminaron el 3 de marzo y el 
examen se realizó el 22 de marzo, es decir, casi 3 semanas más tarde, tiempo suficiente para su preparación.

- Convección: el examen consta de 1 pregunta práctica o problema, donde el alumno puede llevar todo lo que estime oportuno, incluidos libros y ejercicios, siempre que sea en formato papel. Las clases de este bloque terminaron el 7 de abril y el examen se realizó el 27 del mismo mes, es decir, casi 3 semanas más tarde, tiempo suficiente para su preparación.

\section{RESUltados}

El primer resultado es el del test de control de homogeneidad. Para evaluar dicho test de 5 preguntas basadas en lo enseñado en la clase 1, se consideran sólo las respuestas buenas sin penalización de las respuestas incorrectas. En dicho test, el resultado obtenido con la prueba no paramétrica de U de Mann-Whitney o Mann-Whitney-Wilcoxon es que no se puede rechazar la hipótesis nula que supone que las dos muestras (las notas de los grupos GIE1 y GIE2) de distribuciones continuas tienen la misma mediana para un valor de significación de 0,05. El valor p obtenido es 0,513 y por tanto, mayor que 0,05 . Sin embargo, el valor medio sobre 10 para el grupo de control o GIE 1 es de 5,35 con desviación estándar de 2,63, mientras que en el grupo experimental o GIE2 la media es 4,92 y la desviación 2,54. Esto significa que no podemos decir que los grupos son diferentes y por tanto al aplicar la metodología cualquier cambio es demostrativo, sin embargo, parece que el GIE1 parte con cierta ventaja a nivel medio.

Seguidamente podemos extraer de esta experiencia las notas obtenidas por los alumnos de los dos grupos, GIE1 y GIE2, en los exámenes parciales de conducción y convección. En la Tabla 1, el p-valor corresponde a la prueba no paramétrica de U de Mann-Whitney o Mann-Whitney-Wilcoxon sobre una cola, donde la muestra GIE2 se comprueba si es más alta que GIE1, siendo la hipótesis nula la igualdad en las medianas de ambas muestras. En dicha tabla se observa que, aunque las diferencias no son significativas para un valor de significancia de 0,05 y que por tanto no podemos decir que las medianas sean significativamente diferentes, sí lo serían para un valor de 0,1 en el caso de la prueba de conducción. A pesar de esto, como tendencia se observa que las notas son mayores en el grupo experimental que en el grupo de control, invirtiéndose la tendencia vista en el test de homogeneidad, donde el GIE1 presentaba mejores resultados. Las desviaciones en la media también son menores en el grupo experimental para ambos bloques, y esta menor dispersión refleja que el grupo es más homogéneo en su aprendizaje. Además, se observa como el alumnado se presenta sistemáticamente más al examen en el grupo experimental (GIE2), donde no baja del 90\% de asistencia al examen, que en el grupo de control (GIE1), donde en promedio la asistencia al examen es un $17 \%$ menor. Junto con esto, podemos observar que el porcentaje de aprobados frente a presentados es considerablemente superior (A/P en Tabla 1) en el grupo experimental para ambos bloques. En el caso de conducción la diferencia es de un 13\% mientras que en el bloque de convección es de un 10\%; con esto queda patente que el nivel medio del grupo experimental es superior al de control.

En cuanto a la asistencia en clase, en la Figura 2 se muestra el porcentaje de alumnos que han asistido a 5 o más clases de cada parte de la materia: conducción o convección. Se elige el corte de 5 clases puesto que sería un 55\% del bloque que consta de 9 sesiones. Se puede observar como al principio del curso (conducción) los alumnos asisten en un elevado porcentaje siendo muy importante la asistencia en el GIE2. A medida que avanza el curso, y por tanto, aparecen los exámenes continuos y algunas festividades (semana santa) hace que la asistencia decaiga. También puede deberse al tipo de examen realizado, y es que en convección al ser un examen basado en un solo problema o ejercicio siempre de índole práctica, el alumno considera que no es tan importante asistir a clase puesto que la explicación teórica la tiene en los vídeos. Sin embargo, el examen de conducción al tener preguntas teórico-prácticas despierta la necesidad en el alumno de asistir a la explicación en clase de esos ejercicios. Como se ve en la Tabla 2 los alumnos visualizan los videos de ambos bloques de manera más o menos similar, aunque emplean más tiempo en ver los de convección. El tiempo de reproducción media de los videos de conducción es de un $67 \%$ mientras que los de convección llegan al 78\%. Conviene destacar que el vídeo 6 de convección tiene menos visitas que los demás, pero seguramente es debido a que explica el mismo contenido que el vídeo 5 de conducción, aunque por diferente docente. Además, la sensación recibida de parte de algunos alumnos es que la parte de convección es más continuada y si pierden alguna clase retomar el nivel es complicado.

Tabla 1. Resultados de los exámenes de los dos grupos. Notar que DS es desviación estándar, P/M es el ratio entre presentados y matriculados, y A/M es el ratio entre aprobados y presentados.

\begin{tabular}{lcccc}
\cline { 2 - 5 } & \multicolumn{2}{c}{ Conducción } & \multicolumn{2}{c}{ Convección } \\
\cline { 2 - 5 } & GIE 1 & GIE 2 & GIE 1 & GIE 2 \\
\hline Media & 4,88 & 5,55 & 4,18 & 4,50 \\
(DS) & $(2,96)$ & $(2,52)$ & $(2,89)$ & $(2,80)$ \\
\hline P/M (\%) & 79 & 94 & 68 & 91 \\
\hline A/P (\%) & 51 & 64 & 37 & 47 \\
\hline p-valor & \multicolumn{2}{c}{0,07} & \multicolumn{3}{c}{0,23} \\
\hline
\end{tabular}

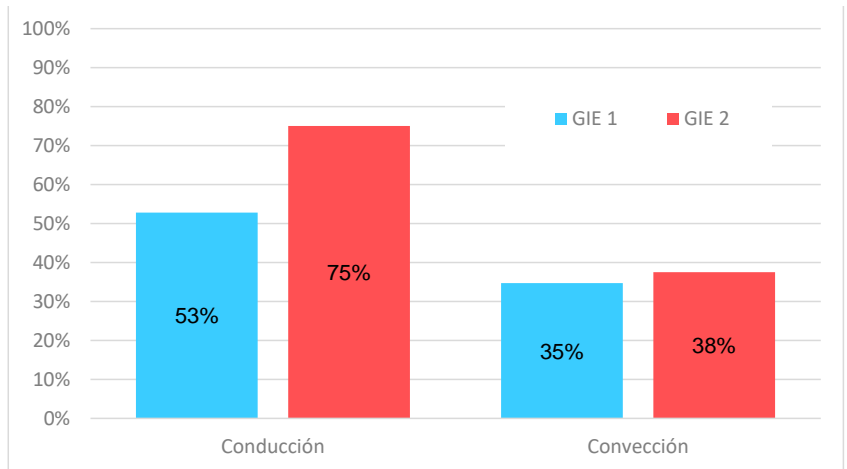

Figura 2. Porcentajes de asistencia a más de 5 clases en ambos bloques.

El número total de visualizaciones de los vídeos para ambos bloques es de 2189 (datos extraídos de Youtube), lo que significa que algunos de los videos son visualizados más de una vez, repartidos entre ordenadores (1642), móviles (306) y tabletas (214). Como se puede apreciar en los números, los videos son vistos preferentemente en el ordenador, probablemente, el alumnado lo haga en el entorno tranquilo de su domicilio, mientras que sólo el $25 \%$ los visualiza en dispositivos móviles. Esto puede deberse a que los recursos 
generados por su extensión (vídeos entre 5 y 10 minutos) y contenido (densidad de información) no sean óptimos para un dispositivo móvil.

Tabla 2. Numero de visualizaciones (A) y tiempo medio de reproducción en porcentaje sobre el total de cada video (B). Nota: la clase 2 de conducción se compone de dos videos.

\begin{tabular}{ccccc}
\cline { 2 - 5 } Clase \# & \multicolumn{2}{c}{ Conducción } & \multicolumn{2}{c}{ Convección } \\
\cline { 2 - 5 } $\mathbf{1}$ & - & - & 218 & 71 \\
\hline $\mathbf{2}$ & 266 & 67 & 169 & 71 \\
\hline $\mathbf{3}$ & 166 & 60 & 148 & 82 \\
\hline $\mathbf{4}$ & 179 & 59 & 129 & 84 \\
\hline $\mathbf{5}$ & 119 & 67 & 105 & 86 \\
\hline $\mathbf{6}$ & 168 & 62 & 95 & 73 \\
\hline $\mathbf{7}$ & 110 & 74 & 116 & 80 \\
\hline $\mathbf{8}$ & 95 & 78 & 106 & 74 \\
\hline Totales & 1103 & 67 & 1086 & 78 \\
\hline
\end{tabular}

Finalmente la tendencia de visualización de los videos por parte del alumnado se ve bien reflejada en la Figura 3. Como puede verse el primer gran pico corresponde a las visualizaciones antes de la clase 1 del 7 de abril de 2017 . Después de esta clase se ven algunas visualizaciones achacadas a gente que comienza el estudio. Después decrecen las visualizaciones, pero se observa un repunte durante la semana santa terminada el 18 de abril de 2017. A partir de esta fecha y hasta el examen (día 27 de abril) se observa una tendencia alcista de las visualizaciones, para evidentemente llegar a cero después del examen.

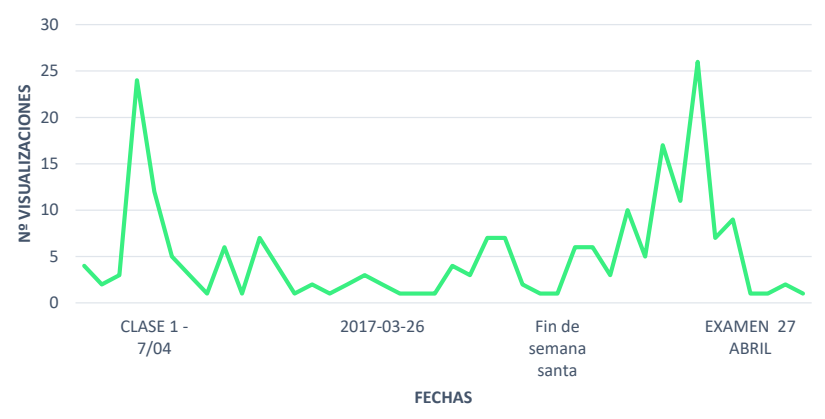

Figura 3. Numero de visualizaciones del vídeo correspondiente a la clase 1 de convección.

Para medir la percepción del alumnado sobre su carga de trabajo, se les realizó un test tipo SEEQ con numerosas preguntas en las que destacamos las siguientes: 1) La carga de trabajo del curso, comparado con otros es ... (1: muy pequeña; 5: muy grande); 2) En promedio, la carga personal de trabajo dedicado a esta asignatura por semana es ... (1: de 0 a 2 h; 2 : de 2 a 4 h; 3: de 4 a 6 h; 4: de 6 a 8 h; 5: más de 8 h). En el grupo de control, de 24 respuestas sobre la pregunta 1, 18 (el $75 \%$ ) indicó que la carga de trabajo estaba en la media (respuesta 3), con un $12 \%$ de alumnos diciendo que estaba por encima de la media (respuesta 4 ) y sólo un $4 \%$ diciendo que estaba muy por encima de la media (respuesta 5). En este mismo grupo, las respuestas sobre la carga de trabajo fuera del aula se repartieron por igual entre 2-4 y 4-6 h, cada una con un $35 \%$, un $22 \%$ reconoció no trabajar más de 2 h a la semana, mientras que tan sólo el $8 \%$ indicó que trabajaba de 6 a 8 h, y nadie indicó que trabajase más de 8 h. En el grupo experimental, de 30 respuestas sobre la pregunta 1, el 67\% (20 respuestas) indicó que la carga de trabajo era igual a la media del curso (de nuevo repuesta 3), sin embargo, un 20\% más indicó que la materia requería de más trabajo (respuesta 4) y un $7 \%$ de mucho más trabajo (respuesta 5). En cuando a la segunda pregunta, un $43 \%$ y un $40 \%$ seleccionaron las respuestas 2 y 3, respectivamente. Sin embargo, un 10\% dijo trabajar menos de $2 \mathrm{~h}$ a la semana, sólo el 7\% indicó trabajar entre 6 y 8 h, y nadie indicó trabajar más de 8 h. Con estos datos podemos afirmar que la gran mayoría del alumnado considera que el trabajo necesario para la materia está dentro del nivel medio del grado, tanto en el GIE1 como en el GIE2. Pero, como se suele indicar en numerosos trabajos con aula invertida (Fidalgo-Blanco, Martinez-Nuñez, Borrás-Gene y Sanchez-Medina, 2017; O'Flaherty y Phillips, 2015), la sensación de parte de los alumnos (alrededor de un 10\%) es que la carga de este curso es grande o muy grande comparada con los demás (respuestas 4 y 5 , respectivamente). Sin embargo, en cuanto a la carga personal de trabajo dedicada el resultado es muy similar en ambos grupos con el 75\% (GIE1) y el $83 \%$ (GIE2) entre 2 y 6 h a la semana. Pero aquí se ve un dato curioso, y es que el uso del aula invertida parece arrastrar a más alumnos (alrededor de un $10 \%$ ) a dedicar más horas por semana que el método tradicional, y ésta puede ser una de las razones por las que los resultados del grupo experimental son mejores que el grupo de control.

\section{CONCLUSIONES}

El modelo aquí aplicado en el global de una materia obligatoria con un gran número de alumnos (82 en el grupo experimental y 152 en el total), demuestra que el aula invertida se puede aplicar con razonable éxito en materias similares. Este modelo no requiere demasiado esfuerzo para el profesorado si la materia está bien organizada y estructurada.

Hoy en día, las escuelas de ingeniería deben producir graduados que sean capaces de resolver problemas, que sean capaces de aprender por sí mismos y que tengan un buen conocimiento técnico. La clase invertida puede jugar un papel fundamental dejando más tiempo para los alumnos centrados y haciéndoles independientes en su estudio. Sin embargo, los resultados serían mejores si en el marco de Bolonia, un mayor número de materias empleasen metodologías que requieran del trabajo del alumno como parte activa de su propio aprendizaje. Un problema detectado en el desarrollo de esta actividad, es el malestar de cierto alumnado por la carga de trabajo diario (en horas y/o estudio) que esta metodología supone para un curso de esta magnitud. Quizá debido a la estructura de nuestro sistema educativo y nuestra sociedad, el uso de estas técnicas requiera de un poco más de tiempo o de un uso menos extremo como el aquí aplicado.

Para paliar uno de los puntos débiles de esta metodología, como es la unión entre actividad online y presencial (Strayer, 2012; Sein-Echaluce, Fidalgo-Blanco y García-Peñalvo, 2015), se ha desarrollado una actividad enlace consistente en la explicación por parte del profesorado de los temas que más errores presentan en la realización de los test o cuestionarios. Esto a su vez es una limitación, puesto que, si los alumnos no participan en gran número y en tiempo para las clases, el uso de esta técnica está limitado ya que es imposible extraer una tendencia en las respuestas del alumnado y por tanto, saber dónde presentan más problemas. Esta técnica puede ser muy útil si por tiempo, el profesor puede extraer un dato individual 
de las respuestas del alumno, para posteriormente en clase atender las carencias individualmente.

En cuanto a los resultados de los exámenes parciales de la materia, aparentemente el aula invertida funciona mejor cuando el examen combina de alguna manera teoría y práctica (conducción) que cuando es básicamente práctica (convección) lo que implica que los conceptos teóricos han sido aprendidos. Esto es un gran aporte pues pocos estudios pueden probar los resultados obtenidos en diferentes tipos de pruebas. Otra limitación encontrada a la vista de los resultados obtenidos, es que los resultados del grupo experimental, GIE2, aunque son ligeramente diferentes al grupo de control (GIE1) en el bloque de Convección, presentan menor diferencia que en conducción posiblemente debido a que la asistencia a clase se iguala en esta parte. Sin embargo, en conducción la diferencia es casi de un $20 \%$ más entre los dos grupos y esto se refleja en la nota media de la clase que es casi 0,7 puntos más alta. Esto viene a reafirmar lo que sugieren algunos autores (Fidalgo-Blanco, Martinez-Nuñez, Borrás-Gene y Sanchez-Medina, 2017) que la clase magistral no debe desaparecer por completo y hay que introducir algo de tiempo en el aula para esta actividad magistral (los link entre cuestionarios on-line y clases), además de los problemas resueltos, sirva al alumno para asentar los conocimientos que adquiere de manera autónoma.

\section{AGRADECIMIENTOS}

Los autores quieren agradecer a la Universidad Politécnica de Madrid (UPM) la financiación aportada a través del proyecto "Aula invertida para la mejora del aprendizaje aplicado a la asignatura de Transferencia de Calor $y$ Materia" con código IE1617.0600 de la convocatoria competitiva "Ayudas a la innovación educativa y a la mejora de la calidad de la enseñanza - 2016” de la UPM.

\section{REFERENCIAS}

Coates, H. (2006). Student engagement in campus-based and online education: University connections. Routledge.

Cortés Martín, J. M. (2010). El aprendizaje basado en problemas como técnica didáctica del Derecho Internacional Público. Docencia y Derecho, (2).
Fidalgo-Blanco, A., Martinez-Nuñez, M., Borrás-Gene, O., \& Sanchez-Medina, J. J. (2017). Micro flip teaching-An innovative model to promote the active involvement of students. Computers in Human Behavior, 72, 713-723.

Mason, G. S., Shuman, T. R., \& Cook, K. E. (2013). Comparing the effectiveness of an inverted classroom to a traditional classroom in an upper-division engineering course. IEEE Transactions on Education, 56(4), 430435.

Medina, J. (2008). Brain rules: 12 principles for surviving and thriving at work, home, and school. Pear Press.

O'Flaherty, J., \& Phillips, C. (2015). The use of flipped classrooms in higher education: A scoping review. The Internet and Higher Education, 25, 85-95.

Sanchidrián, J. A. (2014). Transferencia de Calor. $2^{\circ}$ ed, Fundación Gómez-Pardo, E.T.S.I. Minas y Energía Universidad Politécnica de Madrid.

Sein-Echaluce, M. L., Fidalgo-Blanco, A., \& García-Peñalvo, F. J. (2015). Metodología de enseñanza inversa apoyada en b-learning y gestión del conocimiento. La Sociedad del Aprendizaje. En Actas del III Congreso Internacional sobre Aprendizaje, Innovación y Competitividad. CINAIC (pp. 464-468).

Soler, E. Núñez, J.C., González-Pienda J.A. Álvarez, L. (coords.) (2002). Estrategias de aprendizaje: concepto, evaluación e intervención. Psicología. Sección Pedagogía. Madrid: Pirámide.

Strayer, J. F. (2012). How learning in an inverted classroom influences cooperation, innovation and task orientation. Learning Environments Research, 15(2), 171-193.

UNESCO. (2013). Policy guidelines for mobile learning. Disponible:

http://unesdoc.unesco.org/images/0021/002196/219641e. pdf Acceso: 15/05/2017.

Yoshida, H. (2016). Perceived Usefulness of" Flipped Learning" on Instructional Design for Elementary and Secondary Education: With Focus on Pre-service Teacher Education. International Journal of Information and Education Technology, 6(6), 430. 